\title{
WSN Gait Monitoring for Objective Evaluation of Rehabilitation Process
}

\author{
Octavian Postolache ${ }^{1}$, Senior Member IEEE, José M. D. Pereira ${ }^{2,3}$, Senior Member IEEE, \\ Pedro Silva Girão ${ }^{4}$, Senior Member IEEE, Vítor Viegas ${ }^{2,3}$, Gabriela Postolache ${ }^{5}$ \\ ${ }^{1}$ Instituto de Telecomunicações/ISCTE-IUL, Lisboa, Portugal \\ ${ }^{2}$ Escola Superior de Tecnologia de Setúbal do Instituto Politécnico de Setúbal, Setúbal, Portugal \\ ${ }^{3}$ Instituto de Telecomunicações, Lisboa, Portugal \\ ${ }^{4}$ Instituto de Telecomunicações/DEEC-IST, UL, Lisboa, Portugal \\ ${ }^{5}$ Instituto de Medicina Molecular, Lisboa, Portugal \\ Phone: +351-265-790-000, Fax: +351-265-721-869, Email: dias.pereira@estsetubal.ips.pt
}

\begin{abstract}
A survey of wireless sensor network solutions for gait assessment is presented including own developed solution gait assessment based on smart insoles nodes ZigBee compatible characterized by multichannel force measurement and MEMS inertial measurement IMU. The system was developed to measure ground reaction force, acceleration and direction of feet in order to provide information to physiotherapists for an objective evaluation of rehabilitation effectiveness. Based on acquired data from the sensing channels a set of gait feature extraction such as walking speed stride length, swing time are calculated as part of gait analysis. Software for WSN node control, gait feature calculation and primary analysis of rehabilitation effectiveness developed for the physiotherapist was designed and implemented and preliminary tests were carried out for normal and simulated anomalous gait.
\end{abstract}

Keywords - wireless sensor network, piezoresistive force sensors, inertial measurements, gait analysis.

\section{INTRODUCTION}

Clinical or quantitative gait analysis is a topic of paramount importance in which concerns the detection of gait abnormalities and the support of decision-making relating to people that are under physiotherapeutic treatments. It is important to obtain quantitative data regarding the measurement of forces and walking kinematic parameters like position, velocity and acceleration. There exist a large number of techniques that can be used for gait assessment. Image based techniques are powerful to recognize and identify gait abnormalities and to evaluate patients' evolution over physiotherapy treatments. However, these techniques are affected by the subjectivity of the observers that judge the quality of gait and by their accumulated knowledge and experience in the area. By its turn, even if there exist videography based techniques to perform kinematic analysis, these techniques, usually based on the usage of reflective markers, are also prone to errors related with the image accuracy and resolution that depends on the type of markers that are used and on the contrast between them and the surroundings. Repeatability errors caused by variations in the positioning of the markers are also a great disadvantage of these methods. Another type of techniques that can be used for gait assessment are based on electromyography (EMG). In this case, EMG electrodes acquire voltage signals that give information about muscles' activations. However, these signals have a very low amplitude being required an adequate signal conditioning to obtain an acceptable signal-to-noise ratio of the signal before digitalization or recording. The electrodes pick up electrical noise and the electrodes' connecting wires behave like antennas that cause additional problems. Moreover, non-invasive surface electrodes only gives a gross estimation of muscle activity with low resolution. Even if it is true that every technique has its own advantages and disadvantages, in this paper quantitative techniques based on the usage of accelerometers [1-2], gyroscopes [3-4], inertial measurement units [5-7] and force sensing units [8] are considered. Transmission forces, body accelerations and direction, and other physical variables related with ground reaction forces are measured and processed, in a real-time mode, in order to extract gait related parameters. As a result of the present work, a low-cost, noninvasive and wireless system for gait monitoring was implemented and tested with successful results.

This paper is organized as follows: Sections II and III present the system's description, namely, its hardware and software parts, respectively; Section IV presents experimental results and data analysis and Section V presents paper's conclusions.

\section{CONCLUSIONS}

The paper presented a low-cost, non-invasive and wireless system for gait monitoring. Kinematic parameters related with acceleration and direction are obtained using an inertial measurement unit. Ground reaction forces are also accessed by using smart insoles that include, each one, a set of three force sensing resistors. The basic configuration of the system includes a coordinator node, the gait assessment base station, and two terminal nodes, each one in each leg of the subject under gait analysis, wirelessly interconnected. Several 
algorithms to extract, in time and in frequency domains, information about temporal, linear and angular movements were also developed. From the testing results it can be concluded that the proposed system represents a suitable solution to identify normal and abnormal gait patterns and to provide reliable information to physiotherapists concerning an objective evaluation during rehabilitation processes.

\section{ACKNOWLEDGMENT}

This work was supported by the Instituto de Telecomunicações and Fundação para a Ciência e Tecnologia - project EHR-Physio: Electronic Health Records-Needs, Requirements and Barriers in Physiotherapy, PTDC/DTPDFS/1661/2012. Additional support for performed research was provided by IT-IUL/ISCTE-IUL.

\section{REFERENCES}

[1] J. Cancela, M. Pastorino, M. T. Arredondo, O. Hurtado, "A telehealth system for Parkinson's disease remote monitoring. The PERFORM approach", 35th Annual International Conference of the IEEE EMBS, pp. 7492-7495, July 2013.

[2] E. Sejdić, K.A. Lowry, J. Bellanca, M. S. Redfern, Jennifer S. Brach, "A comprehensive assessment of gait accelerometry signals in time, frequency and time-frequency domains", IEEE Transactions on Neural systems and Rehabilitation Engineering, vol, 22, no. 3, pp. 603-612, 2014.

[3] A. Salarian, P. R. Burkhard, F. J. G. Vingerhoets, B. M. Jolles, K Aminian. "A novel approach to reducing number of sensing units for wearable gait analysis systems", IEEE Transactions on Biomedical Engineering, vol.60, no.1, pp.72-77, January 2013.

[4] J. Barth, C. Oberndorfer, P. Kugler, D. Schuldhaus, J. Winkler, J. Klucken, B. Eskofier, "Subsequence dynamic time warping as a method for robust step segmentation using gyroscope signals of daily life activities", IEEE EMBS 35th Annual International Conference, pp.6744-6747, July, 2013.

[5] D. Novak, P. Reberšek, T. Beravs, J. Podobnik, M. M. De Rossi, M. Donati, T. Lenzi, N. Vitiello, M.C. Carrozza, "Early recognition of gait initiation and termination using wearable sensors", The Fourth IEEE RAS/EMBS International Conference on Biomedical Robotics and Biomechatronics, pp. 1937-1942, June 2012.

[6] R. S. Hundza, W. R. Hook, C. R. Harris, S. V. Mahajan, P. A. Leslie, C. A. Spani, L. G. Spalteholz, B. J. Birch, D. T. Commandeur, N. J. Livingston, "Accurate and reliable gait cycle detection in Parkinson's disease", IEEE Transactions on Neural Systems and Rehabilitation Engineering, vol. 22, no. 1, pp. 127-137, January 2014.

[7] Juan C. Alvarez, R.C. Gonzável, Diego Alvarez, et al., "Multisensor Approach to Walking Distance Estimation with Foot Inertial Sensing", Proc. of the 29th Annual International Conference of IEEE EMBS, Vol. 1, pp. 5719-5722, 2007.

[8] J.M. Dias Pereira, O. Postolache, Vítor Viegas, Pedro Silva Girão, " A Low Cost Measurement System to Extract Kinematic Parameters from Walker Devices", International Instrumentation \& Measurement Technology Conference 2015 (I2MTC'2015), Pisa, Italy, May, 2015.

[9] Microchip Technology Inc., "MRF24J40MA - 2.4 GHz IEEE Std. 802.15.4 ${ }^{\mathrm{TM}}$, RF Transceiver Module, 2008.

[10] Interlink Electronics, "Force Sensing Resistors: integration guide and evaluation parts catalog", Available at: http://www.interlinkelectronics.com [accessed May 2015].

[11] Frank van der Goes, Gerard Meijer, "A Simple Accurate BridgeTransducer Interface with Continuous Autocalibration", IEEE Trans. Instrum. Meas., vol. 46, no.3, pp. 704-710, June 1997.

[12] Mettler-Toledo Gmbh, "Mettler Toledo Spider 1S Scales", Switzerland, 1999.

[13] Analog Devices, ADG1414 octal SPST, Available at: http://www.analog.com/en/switchesmultiplexers/analog switches/adg1414/products/product.html [accessed May 2015].

[14] Gerard C. M. Meijer, "Concepts and Focus Point for Intelligent Sensor Systems", Sensors and Actuators A, 41-42, pp. 183-191, 1994.

[15] STMicroelectronics, " L3G4200D MEMS motion sensor: ultra-stable three-axis digital output gyroscope", Available at: http://www.st.com/web/en/resource/technical/document/datasheet/CD00 265057.pdf [accessed May 2015].

[16] STMicroelectronics, " LSM303DLHC: Ultra compact high performance e-compass: 3D accelerometer and 3D magnetometer module", Available at: http://www.st.com/web/catalog/sense_power/FM89/SC1449/PF251940 [accessed May 2015].

[17] Atmel, "Atmel 8-Bit Microcontroller with 4/8/16/32kbytes In-System Programmable Flash", Available at: http://www.atmel.com [accessed May 2015].

[18] Sparkfun, "Arduino Fio", Available at: https://www.sparkfun.com/products/10116 [accessed May 2015]. 